\title{
Orientation-specific luminance aftereffects
}

\author{
H. H. MIKAELIAN, M. J. LINTON, and M. PHILLIPS \\ University of New Brunswick, Fredericton, New Brunswick, Canada
}

\begin{abstract}
Prolonged viewing of bright vertical (horizontal) gratings alternating with dim horizontal (vertical) gratings generates negative brightness aftereffects that are contingent on the orientation of orthogonal test gratings. The effect is measured by a brightness cancellation technique, similar to the color cancellation technique used in measuring McCollough effects. Like the latter, brightness aftereffects appear to persist for long periods. The magnitude of these aftereffects is a positive monotonic function of the luminance difference between the inducing gratings, and it depends on the conditions of induction; monocular induction generates larger aftereffects than binocular induction does. The aftereffect transfers interocularly, although its mangitude in the contralateral eye is substantially attenuated; binocular measurement, following monocular induction, results in even smaller aftereffects. An attempt to understand these findings within the computational model of brightness perception developed by Grossberg and Mingolla (1985a, 1985b) is presented.
\end{abstract}

The ability of the visual system to discriminate differences in brightness within a given region of visual space, or between such regions, is one of its most fundamental properties. Slow gradients in luminance distribution yield homogeneous regions of brightness; abrupt differences, especially when bordering such regions, generate edges. Several theoretical models, each with distinct hypothetical mechanisms, are available to account for the processing of such visuospatial information (Campbell \& Robson, 1968; Grossberg, 1984; Wilson \& Bergen, 1979; Wilson, McFarlane, \& Phillips, 1983).

Visuospatial aftereffects generated by selective adaptation procedures have been an effective tool in the probing of mechanisms postulated by the various models, and numerous investigators have used the phenomenon to examine the operation of these hypothetical visual mechanisms. Here we report a series of experiments on aftereffects that pertain to the luminance channel mechanisms of brightness and edge perception. The studies deal with orientation-specific brightness aftereffects that are produced when two alternately presented adapting fields, consisting of orthogonally oriented grids that differ in spaceaveraged luminance, are viewed for a prolonged period of time.

The rationale for these studies is derived from experiments on orientation-specific chromatic aftereffects, or McCollough effects. These aftereffects appear as weak complementary hues on orthogonally oriented achromatic test gratings; they occur when subjects view, for several minutes, alternately presented red and green orthogonal gratings (McCollough, 1965; Stromeyer, 1978). The

This research was supported by a grant from the Natural Sciences and Engineering Research Council of Canada. Reprint requests should be sent to H. H. Mikaelian, Department of Psychology, Bag Service No. 45444, University of New Brunswick, Fredericton, New Brunswick, E3B 6E4 Canada. chromatic aftereffects are said to reflect the output of color-coded oriented units in the cortex, although other explanations have also been offered (Harris \& Gibson, 1968; Murch, 1976; Shute, 1979; Siegel \& Allan, 1985). Since there is some evidence that brightness, in addition to wavelength, may also have spatial linkages (Mayhew \& Anstis, 1972), we were interested in determining whether one could probe for luminance-coded orientation units by attempting to generate orientation-specific brightness aftereffects; if such aftereffects could be generated, they would appear as differences in brightness between two orthogonally oriented test gratings equal in spaceaveraged luminance (and in contrast).

Brightness aftereffects contingent on orientation have been reported previously by Over, Broerse, Crassini, and Lovegrove (1974), and more recently by Allan and Tirimacco (1987). To induce the aftereffect, Over et al. (1974) used alternately presented vertical and horizontal gratings (1 cpd) that differed (by a factor of about 46) in averaged luminance. Aftereffects were tested by matching the brightness of vertical and horizontal test gratings. The results indicated, in general, that if, during the 30min induction phase, a dim vertical grating was viewed in alternation with a more luminous horizontal grating, then vertical-horizontal test gratings that were equal in averaged luminance appeared to differ in brightness; to achieve a brightness match, the vertical grating was made darker than the horizontal. Reversing the orientationluminance contingencies of the inducing stimuli resulted in a brightness match when the vertical test grating was made more luminous than the horizontal. In addition, when induced monocularly, the aftereffect did not transfer to the contralateral eye. It seemed, therefore, that brightness aftereffects could be generated, and also that these were much like McCollough effects. These findings led Over et al. (1974) to conclude that there are detectors in the human visual system that are conjointly tuned 
to luminance and contour orientation, and that, like their wavelength-spatial relative, they serve monocular channels.

While Over et al. (1974) may have observed brightness aftereffects, it is likely that these effects were contaminated by afterimages that may have been produced during induction. The space-averaged luminance of the bright pair of the high-contrast inducing square-wave grating was $445 \mathrm{~cd} / \mathrm{m}^{2}$, which calculates to a luminance of approximately $800 \mathrm{~cd} / \mathrm{m}^{2}$ for the bright bar of that grating. At a spatial frequency of $1 \mathrm{cpd}$, such a stimulus is low enough in frequency, and bright enough, so that viewing it in alternation for $\mathbf{3 0}$ min could, among other things, generate strong afterimages. Judgments about aftereffect magnitude could then have been contaminated by the presence of these afterimages, since the low spatial frequency of the test grating would have facilitated juxtaposition of the test stimulus with the afterimage. Failure of interocular transfer further attests to the possibility that afterimages, which are monocular, may have been partially responsible for the observed monocularity of the induced aftereffect.

It should be possible to generate brightness aftereffects and measure their magnitudes with less contamination by afterimages. This may be effected by (1) using pairs of inducing stimuli, the more luminous of which is not too intense, (2) frequently reminding the subject to scan the inducing stimuli (to reduce preferential fixation of contours), and (3) using higher spatial frequency inducing and test gratings (closer to that of chromatic gratings used in McCollough effects). These conditions will make it less likely that afterimages, if any are generated, could be readily juxtaposed on the test stimulus and thereby contaminate the brightness aftereffect. The following experiments were conducted to explore the issue.

\section{EXPERIMENT 1}

Our purpose in the first experiment was to determine whether orientation-specific brightness aftereffects could be induced by alternate viewing of orthogonally oriented gratings that differed in luminance, but with the brighter grating significantly less luminous than that used by Over et al. (1974). A second purpose was to examine whether the magnitude of luminance differences between the alternately viewed inducing gratings was a significant factor in establishing the extent of the aftereffect.

\section{Method}

Subjects. Thirty-five undergraduate students with normal or corrected-to-normal vision were used as observers.

Apparatus and Stimuli. The inducing stimuli consisted of achromatic vertical and horizontal square-wave gratings alternately projected on a rear-projection screen. The spatial frequency of the gratings was $3 \mathrm{cpd}$, with a stimulus field size of $6^{\circ} \times 6^{\circ}$ of visual arc. Seven different pairs of horizontal and vertical gratings (slides) were used, one pair for each inducing condition. One grating in each pair had a space-averaged luminance of $113.1 \mathrm{~cd} / \mathrm{m}^{2}$, while the other grating had any one of the following four luminances: $113.1,56.8,24.1$, or $6 \mathrm{~cd} / \mathrm{m}^{2}$. Each pair was calibrated individu- ally, using neutral density filters. The contrast ratio for each pair of inducing gratings was fixed and maintained at approximately $85 \%$.

The test stimuli were constructed separately and presented on a display adjacent to the inducing screen. They consisted of achromatic horizontal and vertical square-wave grating transparencies, mounted adjacently to each other, so that they could be viewed simultaneously through a porthole in front of the display. The grating transparencies were reproduced photographically on film plates (approximately $13.5 \mathrm{~cm}^{2}$ each) to have a contrast of about $75 \%$ when transilluminated. Each transparency subtended $6^{\circ} \times 6^{\circ}$ of visual arc and had a spatial frequency of $3 \mathrm{cpd}$. The transparencies were sandwiched, along with polarizing filters, between glass plates, with the direction of polarization of the vertical grating filter being orthogonal to that of the horizontal. They were transilluminated and viewed through a polarizing (analyzer) disk, which was mounted on the porthole. The analyzer disk on the porthole could be rotated clockwise or counterclockwise by the observer. Rotating the disk altered the space-averaged luminances of the orthogonal test gratings in opposite directions symmetrically (within the range of luminances used during testing), thereby increasing the luminance of one while decreasing that of the other, without significantly altering their contrast ratio, which remained at about $75 \%$. This was also verified by calculating the contrast ratio after taking careful measurements of the luminances of the dark and the light bars, using a Pritchard spot photometer, with a series of ascending and descending adjustments. The orientation of the analyzer disk, which could be read to within $.1^{\circ}$, was converted into luminance values (described below). With this arrangement, the maximum spaceaveraged luminance of the vertical (horizontal) test grating that could be achieved by rotating the analyzer disk was $53.5 \mathrm{~cd} / \mathrm{m}^{2}$, and the minimum was $8 \mathrm{~cd} / \mathrm{m}^{2}$; corresponding orientations of the analyzer disk for these extreme luminance values were, of course, separated by $90^{\circ}$. An objective match between the vertical and horizontal test gratings existed when the analyzing filter on the disk deviated $45^{\circ}$ from the orientation-generating maximum, or minimum, luminance; at this orientation, which was calibrated to read $0^{\circ}$ on the porthole, the space-averaged luminance of either test grating was $28 \mathrm{~cd} / \mathrm{m}^{2}$. Deviations from $0^{\circ}$ reflected differences in luminance between the orthogonal test gratings; positive values meant higher luminance of the vertical grating, and negative values meant higher luminance of the horizontal grating. A cosine function was used to convert orientation into luminance values (transmission mediated by linearly polarizing filters is described by a cosine function). This arrangement proved to be quite satisfactory for measurement of changes in space-averaged luminance between the two orthogonally oriented test gratings without altering their contrast ratio; as indicated above, the contrast remained constant within the relatively narrow ranges of luminance variation needed to measure the aftereffect.

The absolute value of the aftereffect was determined by calculating the mean difference between pre- and postexposure luminance matches of the horizontal and vertical test gratings. The calculated value represented the amount of luminance added to one of the test gratings and subtracted simultaneously from the other, to cancel the perceived brightness difference induced by the aftereffect between the orthogonal test gratings.

Design. The 35 observers were randomly assigned to one of seven groups, a group being defined by which of the seven pairs of inducing stimuli they viewed during the adaptation phase. For Groups 1,2 , and 3 , the inducing stimuli were arranged in Sequence $A$ (bright horizontal alternating with dim vertical gratings), and consisted of a horizontal grating of $113.1 \mathrm{~cd} / \mathrm{m}^{2}$ space-averaged luminance paired with a vertical grating of $6,24.1$, and $56.8 \mathrm{~cd} / \mathrm{m}^{2}$, respectively. Group 4, the control group, viewed horizontal and vertical gratings of $113.1 \mathrm{~cd} / \mathrm{m}^{2}$ space-averaged luminance each. For Groups 5, 6, and 7, the orientation/luminance contingencies of the inducing stimuli were reversed (Sequence B; see Table 1). 
Table 1

The Space-Averaged Luminances (in $\mathrm{cd} / \mathrm{m}^{2}$ ) of the Inducing Stimuli Used for Each Observer Group

\begin{tabular}{|c|c|c|c|c|c|c|c|}
\hline \multirow[b]{3}{*}{ Orientation } & \multicolumn{7}{|c|}{ Group } \\
\hline & \multicolumn{3}{|c|}{ Sequence A } & \multirow{2}{*}{$\frac{\text { Control }}{4}$} & \multicolumn{3}{|c|}{ Sequence B } \\
\hline & 1 & 2 & 3 & & 5 & 6 & 7 \\
\hline $\begin{array}{l}\text { Horizontal } \\
\text { Vertical }\end{array}$ & $\begin{array}{c}113.1 \\
6\end{array}$ & $\begin{array}{r}113.1 \\
24.1\end{array}$ & $\begin{array}{r}113.1 \\
56.8\end{array}$ & $\begin{array}{l}113.1 \\
113.1\end{array}$ & $\begin{array}{c}6 \\
113.1\end{array}$ & $\begin{array}{r}24.1 \\
113.1\end{array}$ & $\begin{array}{r}56.8 \\
113.1\end{array}$ \\
\hline
\end{tabular}

Procedure. Observers viewed the test stimuli (through the analyzer disk) from a distance of $145 \mathrm{~cm}$, and were instructed to adjust the disk (by rotating it), until the two orthogonal gratings appeared to be equal in brightness. Practice adjustments were allowed until each observer felt comfortable with the task and consistent matches (i.e., matches that varied no more than $3 \%$ in five consecutive trials) were obtained. The experimental session began with six preexposure brightness matches of the test gratings. After each brightness match, the observer was instructed to close the eyes while the experimenter recorded the orientation of the analyzer disk. Disk orientation was changed between each match so that the spaceaveraged luminance of either the vertical or the horizontal grating was higher for the next trial.

The aftereffects were induced as follows: Observers viewed the rear-projection screen from a distance of $190.5 \mathrm{~cm}$ for a period of $20 \mathrm{~min}$, during which the pair of inducing gratings was projected in alternation every $10 \mathrm{sec}$. To reduce the likelihood of developing afterimages, the observers were instructed (and frequently reminded) to scan the gratings and not to fixate any one part. Following the 20-min adaptation period, the subjects shifted their gaze onto the test stimulus display for six postexposure brightness matches of the test gratings.

\section{Results and Discussion}

Absolute values of the aftereffects-that is, mean preand postluminance differences of the brightness matches of the test gratings-were converted to percent change. This percentage was calculated by dividing the absolute value of the aftereffect by the mean preadaptation luminance-that is, the luminance at which, during preexposure measurements, a brightness match existed between the vertical and horizontal test gratings (usually close to $28 \mathrm{~cd} / \mathrm{m}^{2}$ ). Figure 1 shows percent aftereffect as a function of the differences in the luminances between the adapting stimuli (the sign of the scores generated by Groups 5, 6, and 7 was changed to facilitate comparison). The data are replotted in Figure 2 on log-linear coordinates.

A $2 \times 15$ (sequence $\times$ subject) analysis of variance (ANOVA) showed no significant differences between the Sequence $A$ and Sequence $B$ results, indicating that the luminance/orientation contingency was not a significant factor $[F(1,28)=0.0024, p>.05]$. The data from Groups 1,2, and 3 were combined with those of the corresponding Groups 5, 6, and 7 for all subsequent analysis. A $4 \times n$ (group $\times$ subject) ANOVA showed a significant group factor $[F(3,31)=67.26, p<.001]$. Trend analysis indicated significant linear $[F(1,31)=192.2373$, $p<.0001]$ and quadratic $[F(1,31)=6.0865, p<.02]$ components within the range of stimuli used. Post hoc analysis (Tukey's multiple comparison) showed that all of the groups differed significantly from each other at $p<.05$.

The results show that, following adaptation, a brightness match of the test gratings requires that the horizontal be made more luminous than the vertical if, during adaptation, the horizontal inducing grating was brighter than the vertical. Reversing the contingencies of the inducing stimuli changes the direction of this luminance difference, but not its magnitude. These results confirm the findings reported by Over et al. (1974), and they indicate that orientation-specific brightness aftereffects, resembling orientation-specific chromatic aftereffects, can be generated. It is clear from Figure 1 that aftereffect magnitude is a positive monotonic function of the luminance differences between the inducing gratings (the replotted data indicate a typical straight line psychophysical function). The fact that stronger aftereffects are produced by larger luminance differences parallels a simi-

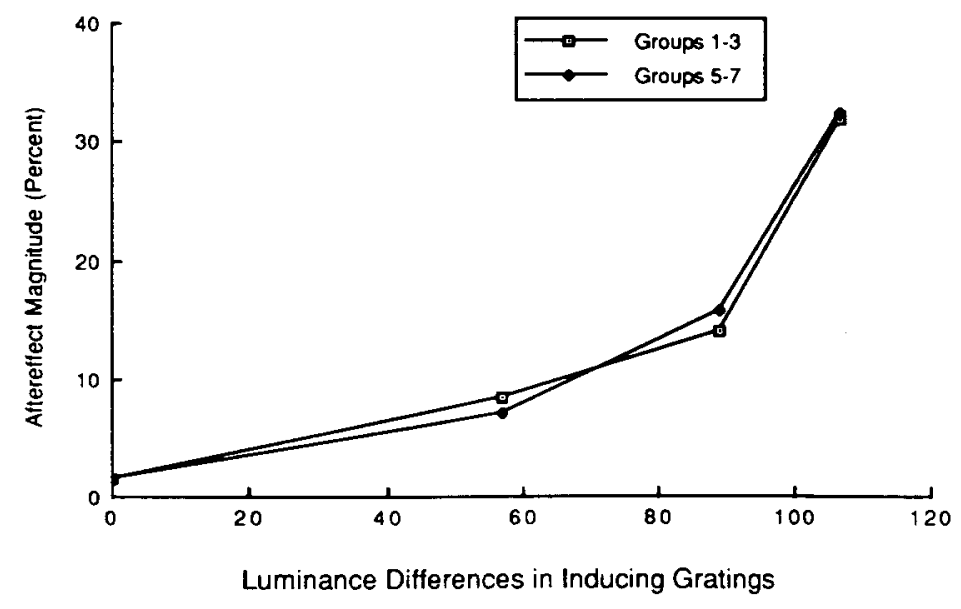

Figure 1. Aftereffect magnitude as a function of luminance differences between the orthogonally oriented inducing gratings. 


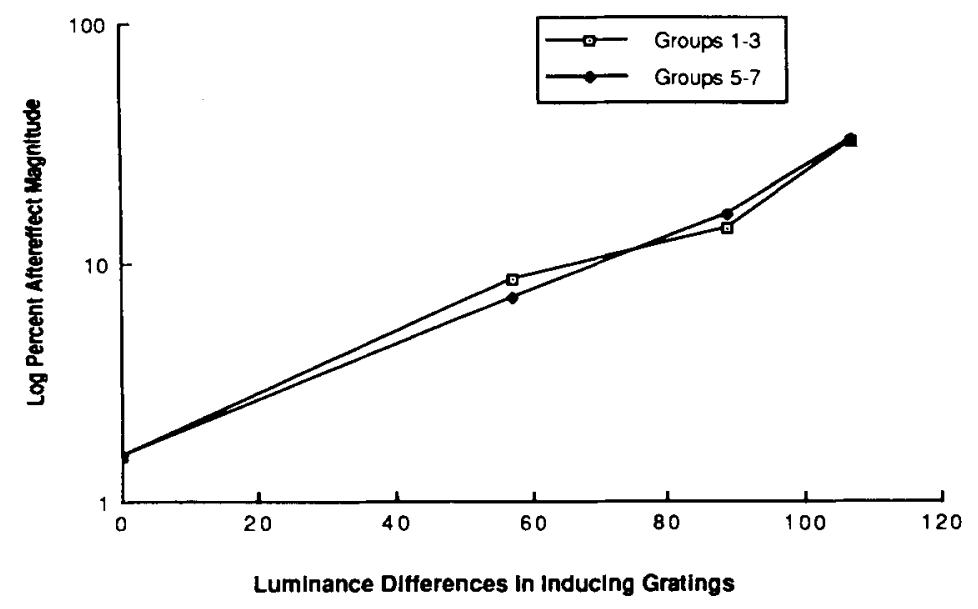

Figure 2. Logarithm of aftereffect magnitude as a function of luminance differences in inducing stimuli.

lar situation in chromatic aftereffects, where the more complementary the inducing colors (the farther they are on the color circle), the stronger the aftereffect (Riggs, White, \& Eimas, 1974). This parallel, however, has to be viewed with caution; one would normally expect stronger stimuli to produce stronger aftereffects. The next experiment demonstrates an important difference between brightness- and wavelength-mediated aftereffects.

\section{EXPERIMENT 2}

One of the well-established characteristics of the luminance channel in the visual system is that it is binocular. Metacontrast, contrast threshold elevation, figural aftereffects, and so forth, all of which are mediated by the luminance channel, manifest robust interocular transfer (Mitchell \& Ware, 1974; Movshon, Chambers, \& Blakemore, 1972; Weisstein, 1971). On the other hand, the chromatic channel tends to process information commonly via monocular pathways. Interocular transfer of many wavelength-mediated phenomena, such as movement aftereffects (Favreau, 1978), chromatic spatial frequency shift (Favreau \& Kavanagh, 1984), and so forth, is not readily observed following monoptic exposure, and it requires additional stimulation of the unexposed eye if it is to occur. This is especially the case for the wavelengthmediated orientation aftereffects, which, apart from some very special circumstances, fail to transfer interocularly (MacKay \& MacKay, 1975; Mikaelian, 1975). As mentioned previously, Over et al. (1974) have reported that under dichoptic viewing conditions, in which the inducing and test stimuli are viewed by different eyes, the brightness aftereffect fails to transfer interocularly. Their observation reflects a curious inconsistency between orientation-specific brightness aftereffects (which obviously are luminance-channel-mediated phenomena and should, therefore, exhibit binocularity) and most other achromatic aftereffects. This apparent monocularity may be an anomalous characteristic of these aftereffects, although a simpler explanation might be that the inducing conditions used by Over et al. (1974) produced afterimages in the ipsilateral eye, which then overwhelmed the detection of the weaker aftereffect in the contralateral eye. Experiment 2 was conducted to determine whether brightness aftereffects, generated by procedures that reduced the likelihood of afterimages, would transfer interocularly.

\section{Method}

Subjects. Twenty naive observers with normal or corrected-tonormal vision were used.

Apparatus and Stimuli. The apparatus was the same as that described for Experiment 1. The space-averaged luminances of the alternately presented inducing gratings were 113.1 and $6 \mathrm{~cd} / \mathrm{m}^{2}$.

Design. The 20 observers were assigned to two groups. The inducing stimuli for Group 1 consisted of the alternately viewed bright $\left(113.1-\mathrm{cd} / \mathrm{m}^{2}\right)$ vertical and $\operatorname{dim}\left(6-\mathrm{cd} / \mathrm{m}^{2}\right)$ horizontal gratings. The brightness/orientation contingencies were reversed (bright horizontal and dim vertical gratings) for Group 2.

Procedure. After an initial training period, monocular brightness matches were obtained with each eye, using the procedures described above. The subjects then viewed the alternately presented inducing stimuli with the dominant eye (determined by sightingdominance test; Porac \& Coren, 1986) for a period of $20 \mathrm{~min}$, while the nondominant eye was covered by an eyepatch. The dominant eye was used during induction because of an earlier observation indicating that it may affect interocular generalization of orientationcontingent aftereffects (Mikaelian \& Staples, 1978). Postexposure brightness matches were obtained with the dominant and nondominant eyes, separately, in a counterbalanced order.

\section{Results and Discussion}

The results are graphed in Figure 3, showing, for the two groups, aftereffect magnitude as percent change from preexposure. For Group 1, the absolute magnitude of the direct effect in the adapted eye is $5.8 \mathrm{~cd} / \mathrm{m}^{2}$, representing a $22.4 \%$ change ( $S E=5.6$ ) from preexposure; in the nonadapted eye, it is $3.01 \mathrm{~cd} / \mathrm{m}^{2}$, an $11.6 \%$ change ( $S E$ $=2.6$ ). These results reflect about $52 \%$ interocular trans- 


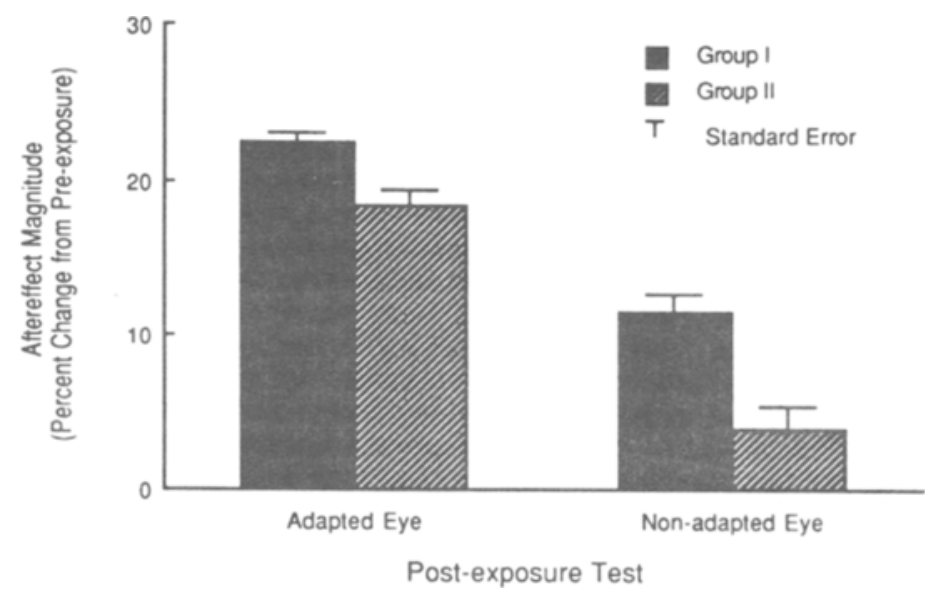

Figure 3. Aftereffect magnitude following monocular induction in the ipsilateral (adapted) and contralateral (nonadapted) eye.

fer. The corresponding figures for Group 2 are: adapted eye, $4.76 \mathrm{~cd} / \mathrm{m}^{2}$, which represents $18.0 \%$ change $(S E=$ 2.3 ); nonadapted eye, $1.03 \mathrm{~cd} / \mathrm{m}^{2}$, a $4.0 \%$ change ( $S E=$ $3.2)$, reflecting about $22 \%$ interocular transfer. A $2 \times$ $10 \times 2$ (group $\times$ subject $\times$ eye) ANOVA revealed eye to be the only significant factor $[F(1,18)=15.06$, $p<.01]$. As shown by $t$ tests on the data (collapsed across groups), aftereffect magnitude measured with the adapted eye, as well as that obtained with the contralateral eye, was significant $[t(19)=12.01, p<.001$, and $t(19)$ $=2.88, p<.01$, respectively $]$.

Contrary to the findings reported by Over et al. (1974), these results indicate that the orientation-contingent brightness aftereffects, when induced monoptically, do transfer to the contralateral eye, and that the magnitude of the transfer may be as high as $50 \%$. The fact that the unadapted eye was covered with an eyepatch during monocular exposure, thus allowing considerable dark adaptation, may have influenced the variability in these results.

Interocular transfer of these aftereffects is consistent with other aftereffects that are mediated by the luminance channel, and it reflects a difference between orientationcontingent brightness aftereffects and similarly contingent chromatic aftereffects.

\section{EXPERIMENT 3}

One of the salient characteristics of the McCollough effect is its longevity. In contrast with aftereffects mediated by the luminance channel, such as contrast threshold elevation or figural aftereffects, where one is hard pressed to measure the aftereffect before it decays exponentially seconds after induction (Magnussen \& Johnsen, 1986), $10 \mathrm{~min}$ of viewing the colored inducing gratings generate chromatic aftereffects that may last for a week or more (Riggs et al., 1974). The next experiment was designed to explore this aspect of the orientation-contingent brightness aftereffect. In addition, in earlier pilot studies for Experiment 2, differences in the magnitude of aftereffects were noted during monocular as opposed to binocular induction of the aftereffect among the subjects. Experiment 3 was devised to enable simultaneous investigation of this incidental observation in a within-subjects design.

\section{Method}

Subjects. Six naive observers with normal or corrected-to-normal vision were used.

Apparatus and Stimuli. The apparatus was the same as that used in Experiments 1 and 2. The inducing stimuli consisted of bright $\left(113-\mathrm{cd} / \mathrm{m}^{2}\right)$ vertical and $\operatorname{dim}\left(6-\mathrm{cd} / \mathrm{m}^{2}\right)$ horizontal gratings.

Design. All 6 observers participated in two different sessions (Session 1 and Session 2) separated by at least $24 \mathrm{~h}$. The order of monocular and binocular exposure was counterbalanced. For 3 of the subjects, induction and tests were done monocularly (dominant eye) during Session 1 and binocularly during Session 2. For the remaining observers, induction and tests were done binocularly during Session 1 and monocularly during Session 2.

Procedure. Training, induction, and test procedures were as described previously. Each session consisted of preexposure luminance matches (upon completion of training) followed by $20 \mathrm{~min}$ of viewing the inducing gratings. Following induction, a series of aftereffect measures was obtained in seven different blocks, with each block of measurements separated by an interval of $3 \mathrm{~min}$ in the dark (plus the $30-\sec$ test duration).

\section{Results and Discussion}

The results are graphed in Figure 4, showing aftereffect magnitude (percent change) as a function of time (test block). The two curves refer to monocular (open symbols) and binocular (solid symbols) conditions of induction and test. A multivariate analysis of variance (MANOVA) showed all postexposure measurements to be significantly different from preexposure measures $[F(1,5)=39.70, p<.005]$, and the monocular measures to be significantly larger than the binocular $[F(1,5)=$ $18.97, p<.01]$. There are no significant differences between the different test blocks. The data clearly show that once the aftereffect is established, it remains essentially unabated for more than $24 \mathrm{~min}$. A series of seven blocks of measurements (each block consisting of at least six adjustments), obtained following induction, seems to 


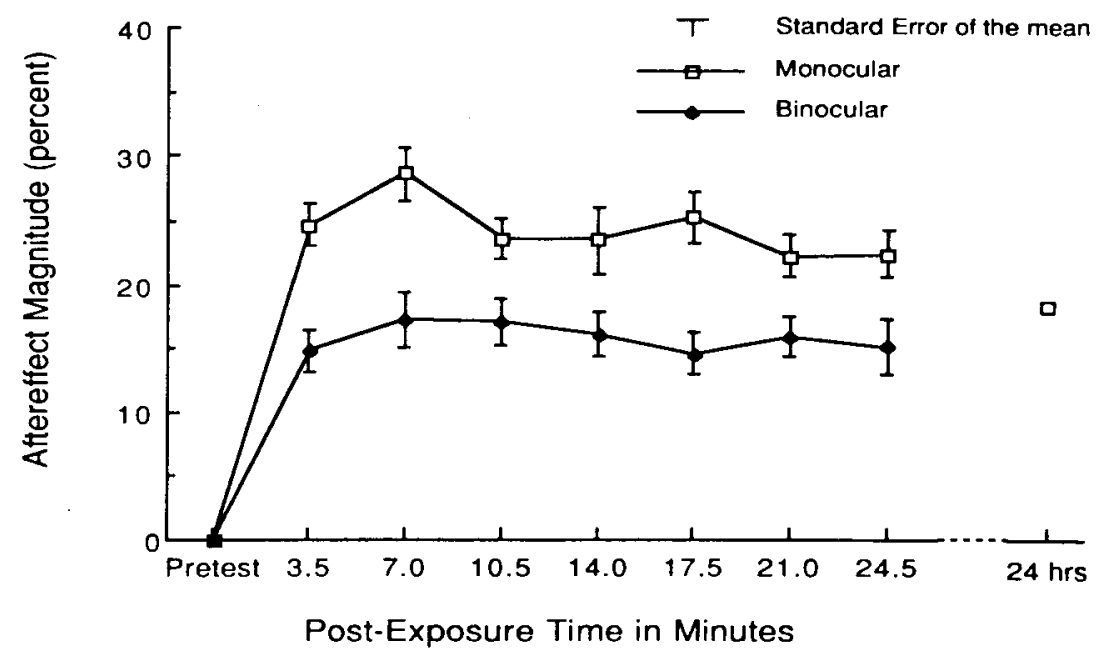

Figure 4. Duration of aftereffect magnitude following monocular and binocular induction.

produce no significant attenuation of the aftereffect. Three of the participants tested (monocularly) $24 \mathrm{~h}$ after Session 2 still manifested reliable aftereffects. In terms of duration, therefore, the orientation-contingent brightness aftereffects appear to parallel the similarly contingent chromatic aftereffects. The duration data also argue against significant contamination of the aftereffect by afterimages, since the latter would have decayed almost entirely toward the end of the testing period. (A note of caution in interpreting the duration data: Repeated measurement of aftereffect magnitude may have, at least partially, contributed the apparent long-term duration.)

An equally clear finding-and one that is no less intriguing-is the reliable difference in aftereffect magnitude as a function of monocular and binocular induction and test. Aftereffects are significantly larger following monocular viewing of the inducing and test gratings, an asymmetry that is present throughout the testing period. Binocular measurements taken following monocular induction showed a significant attenuation (more than 60\%) of the aftereffect $[t(5)=3.66, p<.02]$. Monocular aftereffect measures following binocular exposure were equivalent to binocular measures. Such an observation suggests the importance of monocular induction in generating the asymmetry. The theoretical significance of this observation is considered in the next section.

\section{GENERAL DISCUSSION}

The results of these experiments confirm the fact that orientation-contingent aftereffects mediated by luminance (in contrast to wavelength) can be generated by prolonged viewing of alternately presented gratings that differ in luminance (instead of color). Unlike McCollough effects, these brightness aftereffects transfer interocularly, indicating mediation at a binocular site. It should be noted, however, that interocular transfer is not as massive as is the case in some of the other luminance-mediated after- effects, where often $70 \%$ of the direct effect may be seen at the unadapted eye (Blake, Overton, \& Lema-Stern, 1981; Mitchell \& Ware, 1974; Movshon et al., 1972). Although this observation must be interpreted with caution because interocular transfer is variable even among observers with normal vision, and because our observers were not tested for stereoblindness, which is known to affect interocular transfer (Mitchell \& Ware, 1974), it has an interesting implication for a theoretical model to be discussed later in this section.

The magnitude of the aftereffect is greater following monocular induction and test than following binocular induction, a difference that persists unaltered after seven blocks of test sessions. Such a finding indicates that the enhanced monocular aftereffect is not a transient phenomenon, and that it could reflect the characteristics of the processes generating the aftereffect. Finally, like its wavelength-mediated relative, these aftereffects have long decay times, in some cases remaining essentially unchanged for periods of up to $24 \mathrm{~h}$ postexposure.

These observations raise several theoretical questions. One could view them as confirming the classical learning model that has been applied by Murch (1976) and others (Leppman, 1973; Mayhew \& Anstis, 1972; Siegal \& Allan, 1985; Skowbo, 1984; Skowbo, Gentry, Timney, \& Morant, 1974) to the explanation of McCollough effects. This approach has been criticized elsewhere (Stromeyer, 1978), and the same criticisms are valid with respect to the application of that model to the present phenomenon. A more acceptable alternative, the one developed by Over et al. (1974), might be to regard these results as evidence for luminance-spatial linkages (Mayhew \& Anstis, 1972) that are similar to the wavelengthspatial linkages thought to be implicated by McCollough effects (Stromeyer, 1978). Such an approach would stipulate the existence of neural units that are orientationally selective and coded for luminance. The exposure condition would selectively fatigue these units, rendering them 
less responsive. During testing, fatigued channels, such as the vertical (in the condition where the inducing stimuli consisted of bright vertical bars), would respond less vigorously, producing the perception that the vertical test bars were dimmer than the accompanying horizontal bars, which then would appear to be brighter. When requested to match brightness, the observer will increase the luminance of the vertical test grating until its brightness equals that of the horizontal.

Although the above analysis represents a plausible interpretation of our results, it has several difficulties. These, in increasing order of importance, are the following: Like other luminance channels, the hypothetical luminance-spatial units would have to be binocularly driven, and the aftereffects should, therefore, transfer interocularly in a massive way, quite like tilt or movement aftereffects, threshold elevation, and so forth. Although the orientation-contingent aftereffects do transfer interocularly, the magnitude of this transfer is rather limited. In addition, the results of Experiment 3 show that aftereffects following monocular induction are significantly larger than those following binocular induction; it is difficult to reconcile this finding with the necessarily implied binocularity of these hypothetical luminance-spatial units. A second issue deals with the rate of recovery from fatigue induced by prolonged stimulation of these units. While luminance-coded units respond vigorously to appropriate stimuli and eventually become fatigued (habituated) with prolonged stimulation (which is presumed to be the process underlying the generation of aftereffects), the decay of this fatigue (recovery from habituation) is a matter of fractions of a second following cessation of stimulation, rather than the many minutes that would be necessary to account for our finding that the aftereffect persists unabated for more than 24 min (and sometimes for $24 \mathrm{~h}$; Blakemore \& Sutton, 1969).

The above arguments are not in themselves adequate for one to reject Over et al.'s (1974) model of luminancecoded units; they only serve to indicate some of its theoretical shortcomings. The major difficulty of that model is the following: Although wavelength-spatial linked units might be the possible mechanisms underlying chromatic aftereffects, where no more than two sets of limited response chromatic opponent processes (red-green and blue-yellow mechanisms) are required to be linked to spatial units to account for McCollough effects, the number of luminance processes linked to spatial units required by the Over et al. scheme will have to be quite large to account for the orientation-contingent brightness aftereffects reported by them as well as those found in the present experiment. There may, in fact, exist such a limited set of luminance-coded opponent processes (all responding to a restricted range of luminances), which interact to mediate the perception of brightness (much as their wavelength-coded relatives do to account for the perception of the entire color spectrum). At present, however, we have no evidence for opponent-process luminance units whose responsivity is restricted to a limited range of luminance values.
Our results can be accounted for more parsimoniously by postulating the existence of a single luminance-brightness mediating system, whose output to oriented edge (contour) processing systems changes as a function of the inducing conditions (history of prior stimulation). An interesting approach in line with this speculation-and one that, perhaps, allows a more predictive understanding of the present findings-is the computational model of brightness perception developed by Grossberg and Mingolla (1985a, 1985b). Grossberg and Mingolla postulate that the elements of the visual system are organized into three subsystems: boundary contour (BC), feature contour (FC), and object recognition (OR). The BC system operates on luminance differences, spatial frequency, and orientation, in order to generate boundaries, or edges, in the visual scene. Once boundaries are established (and before they can become visible), the FC system activates a filling-in process to spread color and/or brightness until regions delineated by the borders are permeated. The OR system sends top-down learned template signals so that boundary structures may be modified (for object completion or perceptual grouping). This model further stipulates that contours (the output of the BC system) in orthogonal orientations, such as vertical and horizontal, are mutually inhibitory. Within the context of the present experiment, viewing bright and vertical bars implies inhibiting the orthogonal horizontal bars (meaning they should appear dimmer); in turn, dimmer horizontal bars imply less inhibition of the bright vertical bars. If one assumes that the FC system has a weighting network to balance its output to the two orthogonal orientations, then the prolonged imbalance in the brightness between the two orthogonal orientations during the exposure phase of our procedure would change this balance, thus changing the output of the BC system, and it would thereby induce the observed negative aftereffects on the orthogonally oriented test contours. Such an aftereffect would, of course, be specific to the orientations affected, and should remain unchanged until the imbalance in the FC system between the vertical/horizontal orthogonality is redressed, perhaps by prolonged exposure to a "proper" set of inducing stimuli (stimuli with opposite orientation-luminance contingency). These theoretical stipulations may be extended to McCollough effects, simply by changing the FC output from brightness to color. It is, of course, well established that McCollough effects persist for a long time, and that they can be weakened (though not permanently obliterated) by viewing inducing gratings whose orientation/color contingency is the exact reverse of the original inducing stimuli (MacKay \& MacKay, 1975; Sigel \& Nachmias, 1975; White, 1976). We are currently examining the role of this procedure in the decay of the orientation-specific brightness aftereffects.

The data on interocular transfer fit neatly within a scheme developed by Wolfe and Held $(1981,1983)$ concerning monocular and binocular channels of visual information processing. These authors have proposed a purely binocular process in the visual system, which is activated only when input from both eyes is present (the 
"and" channel). This channel exists in addition to the other binocular channel (the "or" channel), which is activated when input from either eye is present. There also exist, of course, the purely monocular channels. During monocular exposure, both the monocular and the or channels are activated; aftereffects in the ipsilateral eye therefore represent the pooled contributions of both of these channels. In the contralateral eye, aftereffect magnitude is substantially attenuated (about $50 \%$ ) because it is mediated via the or channels only, the other unexposed monocular channel serving to weaken its magnitude. Binocular measurement taps the adapted or channels, but the magnitude of the aftereffect is reduced, due now to the activation of the and channels, which, along with the other unadapted monocular channel, serve to dilute it; thus interocular transfer and binocular measurements yield comparable aftereffect magnitudes.

The data on monocular as opposed to binocular exposure (Figure 4) present a challenge. Following the above scheme, with binocular exposure the monocular channels as well as the two binocular ones (the or and the and units) should contribute to generating the aftereffect. Reduced aftereffect magnitude, measured binocularly or monoculary, following binocular induction suggests inhibitory interaction between the two binocular channels, perhaps extending into the monocular channels. Perhaps the fact that the dominant eye was used during monocular induction was a contributing factor. It is also possible that much longer exposure might eliminate this difference, indicating that the and channel may have a longer time constant. These and other possibilities are currently being examined.

\section{REFERENCES}

Allan, L. G., \& TiRimacco, N. (1987). An orientation-contingent achromatic aftereffect. Bulletin of the Psychonomic Society, 25, 54-55.

Blake, R., OVerton, R. , Lema-STERN, S. (1981). Interocular transfer of visual aftereffects. Journal of Experimental Psychology: Human Performance \& Perception, 7, 367-381.

Blakemore, C., \& SutTon, P. (1969). Size adaptation: A new aftereffect. Science, 166, 245-247.

Camprell, F. W., Robson, J. G. (1968). Application of Fourier analysis to the visibility of gratings. Journal of Physiology, 197, 551-566.

FAVREAU, O. E. (1978). Interocular transfer of color-contingent motion aftereffects: Positive aftereffects. Vision Research, 18, 841-844.

Favreau, O. E., Kavanagh, P. (1984). Interocular transfer of a chromatic frequency shift: Temporal constraints. Vision Research, 24, 1799-1805.

Grossberg, S. (1984). Outline of a theory of brightness, color, and form perception. In E. Degreef \& J. van Buggenhaut (Eds.), Trends in mathematical psychology (pp. 59-86). Amsterdam: North-Holland.

Grossberg, S., Mingolla, E. (1985a). Neural dynamics and form perception: Boundary completions, illusory figures and neon-color spreading. Psychological Review, 92, 173-211.

Grossberg, S., \& Mingolla, E. (1985b). Neural dynamics of perceptual grouping: Textures, boundaries, and emergent segmentations. Perception \& Psychophysics, 38, 141-171.

Harris, C. S., Gibson, A. R. (1968). Is orientation-specific color adaptation in human vision due to edge detectors, afterimages, or "dipoles"? Science, 162, 1506-1507.
LEPPMAN, P. K. (1973). Spatial frequency dependent chromatic aftereffects. Nature, 242, 411-412.

Mackay, D. M., MACKAY, V. (1975). What causes decay of patterncontingent chromatic aftereffects? Vision Research, 15, 462-464.

Magnussen, S., Johnsen, T. (1986). Temporal aspects of spatial adaptation: A study of the tilt aftereffect. Vision Research, 24, 661-671.

MAYHEW, J. E. W., ANSTIS, S. M. (1972). Movement aftereffects contingent on color, intensity, and pattern. Perception \& Psychophysics, 12, 77-85

McCollough, C. (1965). Color adaptation of edge-detectors in the human visual system. Science, 149, 1115-1116.

Mikaelian, H. H. (1975). Interocular generalization of orientation specific color aftereffects. Vision Research, 15, 661-664.

Mikaelian, H. H. \& STAPLes, C. D. (1978, March). Interocular generalization of contingent chromatic aftereffects as a function of sighting dominance. Paper presented at the meeting of the Eastern Psychological Association, Washington, DC.

MitcheLL, D., WARE, C. (1974). Interocular transfer of a visual aftereffect in normal and stereoblind humans. Journal of Physiology, 236, $707-721$

Movshon, J. A., Chambers, B. E. I., Blakemore, C. (1972). Interocular transfer in normal humans, and those who lack stereopsis. Perception, 1, 483-490.

Murch, G. M. (1976). Classical conditioning of the McCollough effect: Temporal parameters. Vision Research, 16, 615-619.

Over, R., Broerse, J., Crassini, B., \& Lovegrove, W. (1974). Orientation-specific aftereffects and illusions in the perception of brightness. Perception \& Psychophysics, 15, 53-56.

PorAC, C., \& COREN, S. (1986). Sighting dominance and ego-centric localization. Vision Research, 26, 1709-1713.

RigGS, L. A., White, K. D., Eimas, P. D. (1974). Establishment and decay of orientation-contingent aftereffects of color. Perception \& Psychophysics, 16, 535-542.

ShUTE, C. C. D. (1979). The McCollough effect: An indicator of central neurotransmitter activity. London: Cambridge University Press.

SiEgel, S., \& AlLAN, L. (1985). Overshadowing and blocking of the orientation-contingent color aftereffect: Evidence for a conditioning mechanism. Learning \& Motivation, 16, 125-138.

Sigel, C., \& NaChmias, J. (1975). A re-evaluation of curvature-specific chromatic aftereffects. Vision Research, 15, 829-836.

Sкоwво, D. (1984). Are McCollough effects conditioned responses? Psychological Bulletin, 96, 215-226.

Skowbo, D., Gentry, T., Timney, B., \& Morant, R. B. (1974). The McCollough effect: Influence of several kinds of visual stimulation on decay rate. Perception \& Psychophysics, 16, 47-49.

STromeyer, C. F. (1978). Form-color aftereffects in human vision. In R. Held, H. Leibowitz, \& H.-L. Teuber (Eds.), Handbook of sensory physiology: Vol. 8. Perception (pp. 97-142), Berlin: SpringerVerlag.

Weisstein, N. (1972). Metacontrast. In D. Jameson \& L. M. Hurvich (Eds.), Handbook of sensory physiology: Vol. 7/4. Visual psychophysics (pp. 233-272). New York: Springer-Verlag.

WHITE, K. D. (1976). Studies of orientation-specific color aftereffects (Doctoral dissertation, Brown University, 1976). Dissertation Abstracts International, 38, 403B.

Wilson, H. R., \&ERGEN, J. R. (1979). A four mechanisms model for threshold spatial vision. Vision Research, 19, 19-32.

Wilson, H. R., McFarlane, D. K., \& Phillips, G. (1983). Spatial frequency timing of orientation selective units estimated by oblique masking. Vision Research, 23, 873-882.

Wolfe, J., \& HeLd, R. (1981). A purely binocular mechanism in human vision. Vision Research, 21, 1755-1759.

Wolfe, J., HeLD, R. (1983). Shared characteristics of stereopsis and the purely binocular process. Vision Research, 23, 217-227.

(Manuscript received January 23, 1989; revision accepted for publication November 27,1989 .) 